\title{
Penrose limits of non-standard brane intersections
}

\author{
H L $\ddot{u}^{1}$ and J F Vázquez-Poritz ${ }^{2}$ \\ ${ }^{1}$ Michigan Center for Theoretical Physics, University of Michigan, Ann Arbor, MI 48109, USA \\ 2 Physique Théorique et Mathématique, Université Libre de Bruxelles, Campus Plaine CP 231, \\ B-1050 Bruxelles, Belgium
}

Received 10 May 2002

Published 15 July 2002

Online at stacks.iop.org/CQG/19/4059

\begin{abstract}
The non-standard intersection of two 5-branes and a string can give rise to $\mathrm{AdS}_{3} \times S^{3} \times S^{3} \times S^{1}$. We consider the Penrose limit of this geometry and study the supersymmetry of the resulting pp-wave solution. There is a one-parameter family of Penrose limits associated with the orthogonal rotation of the two foliating circles within the two 3 -spheres. Supernumerary Killing spinors arise only when the rotation angle is $45^{\circ}$, for which case we obtain the corresponding light-cone string action that has linearly-realized supersymmetry. We also obtain Penrose limits of other non-standard intersections that give rise to the product of $\mathrm{AdS}_{3}$ or $\mathrm{AdS}_{2}$ and 2-spheres. The resulting pp-waves are supported by multiple constant field strengths.
\end{abstract}

PACS number: 1125

\section{Introduction}

The pp-waves arising from the Penrose limits of $\mathrm{AdS}_{p} \times S^{q}$ spacetime provide simple backgrounds on which to study string and M-theory. The maximally supersymmetric ppwave with a constant 4-form field strength was first constructed in [1] for eleven-dimensional supergravity. Recently, the maximally supersymmetric pp-wave in type IIB supergravity with a constant self-dual 5-form field strength was obtained in [2]. These solutions are actually the Penrose limits of $\operatorname{AdS}_{p} \times S^{q}$ spacetimes with $(p, q)=(4,7),(7,4)$ and $(5,5)$, respectively [3]. In the type IIB case, the light-cone string action for the pp-wave $[4,5]$, is exactly solvable. This makes it possible to study the AdS/CFT correspondence on the level of full string theory in the Penrose limit [5], which has generated considerable interest recently [6-26].

$\mathrm{AdS}_{p} \times S^{q}$ arises as the near-horizon geometry of non-dilatonic branes or intersecting branes, for $(p, q)=(4,7),(7,4),(5,5),(3,3),(3,2),(2,3)$ and $(2,2)$. AdS structure also arises from 'non-standard' intersections, for which the harmonic function $H$ for each brane component depends on the coordinates of the relative transverse space rather than those of the overall transverse space [27-29]. The simplest example is the non-standard intersection of two 5-branes plus a string in the common worldvolume, whose near-horizon geometry is $\mathrm{AdS}_{3} \times S^{3} \times S^{3} \times S^{1}[30]$. 
In section 2, we perform the Penrose limit on the above solution. Since the product space involves two 3-spheres, there exists a one-parameter family of Penrose limits of this solution, parametrized by the rotation of the two foliating $S^{1}$ within the two 3-spheres. In section 3, we study the supersymmetry of the resulting pp-wave solution. We find that there are 16 standard Killing spinors associated with any pp-wave solution, but supernumerary Killing spinors arise only for a specific choice of the parameter, associated with a $45^{\circ}$ rotation of the two $S^{1}$. For this case, there are four supernumerary Killing spinors, which are all independent of the $x^{+}$coordinate. Furthermore, 4 out of the 16 standard Killing spinors are also independent of $x^{+}$. We then obtain the supersymmetric light-cone string action in this pp-wave background. In section 4 , we $T$-dualize the pp-wave solution and show that it is related to the D3-brane with one of the worldvolume coordinates fibred over the six-dimensional transverse space. In section 5, we obtain the Penrose limits of more examples of non-standard intersections which give rise to $\mathrm{AdS}_{2}$ or $\mathrm{AdS}_{3}$. Again, all of these examples have a one-parameter family of Penrose limits. We conclude our paper in section 6.

\section{5-5-1 system and Penrose limit}

The 5-5-1 system is supported by a 3-form field strength in $D=10$. The relevant Lagrangian is given by

$$
\mathrm{e}^{-1} \mathcal{L}=R-\frac{1}{2}(\partial \phi)^{2}-\frac{1}{12} \mathrm{e}^{-\phi} F_{(3)}^{2} .
$$

Since our system involves only the metric, the dilaton and the 3 -form field strength, the results apply to types IIA, IIB and heterotic string theories. The solution is given by [30]

$$
\begin{aligned}
\mathrm{d} s_{10}^{2} & =K^{-3 / 4}(H \widetilde{H})^{-1 / 4}\left(-\mathrm{d} t^{2}+\mathrm{d} x^{2}+K H \mathrm{~d} y_{i}^{2}+K \widetilde{H} \mathrm{~d} \tilde{y}_{i}^{2}\right), \\
F_{(3)} & =\mathrm{e}^{\phi} *\left(\widetilde{H} \mathrm{~d} t \wedge \mathrm{d} x \wedge \mathrm{d}^{4} \tilde{y} \wedge \mathrm{d} H^{-1}\right)+\mathrm{e}^{\phi} *\left(H \mathrm{~d} t \wedge \mathrm{d} x \wedge \mathrm{d}^{4} y \wedge \mathrm{d} \widetilde{H}^{-1}\right) \\
& +\mathrm{d} t \wedge \mathrm{d} x \wedge \mathrm{d} K^{-1}, \quad \phi=-\frac{1}{2} \log [K /(H \widetilde{H})], \quad K=H \widetilde{H},
\end{aligned}
$$

where $H$ and $\widetilde{H}$ are harmonic functions in the relative transverse $y_{i}$ and $\tilde{y}_{i}$ spaces, respectively. The metric can be represented by the following diagram:

\begin{tabular}{c|ccccccccccc} 
& $t$ & $x$ & $y_{1}$ & $y_{2}$ & $y_{3}$ & $y_{4}$ & $\tilde{y}_{1}$ & $\tilde{y}_{2}$ & $\tilde{y}_{3}$ & $\tilde{y}_{4}$ & \\
\hline 5-brane & $\times$ & $\times$ & $\times$ & $\times$ & $\times$ & $\times$ & - & - & - & - & $\tilde{H}$ \\
5-brane & $\times$ & $\times$ & - & - & - & - & $\times$ & $\times$ & $\times$ & $\times$ & $H$ \\
string & $\times$ & $\times$ & - & - & - & - & - & - & - & - & $K$
\end{tabular}

5-5-1 system

We parametrize the coordinates as $\mathrm{d} y_{i}^{2}=\mathrm{d} y^{2}+y^{2} \mathrm{~d} \Omega_{3}^{2}$ and $\mathrm{d} \tilde{y}_{i}^{2}=\mathrm{d} y^{2}+y^{2} \mathrm{~d} \widetilde{\Omega}_{3}^{2}$. The isotropic solution is then given by

$$
H=1+\frac{Q}{y^{2}}, \quad \widetilde{H}=1+\frac{\widetilde{Q}}{\tilde{y}^{2}} .
$$

For simplicity, we take $Q=\widetilde{Q}=\lambda^{2}$. The near-horizon geometry of the solution is $\mathrm{AdS}_{3} \times S^{3} \times S^{3} \times S^{1}$ [30]. Expressing the $\mathrm{AdS}_{3}$ in global coordinates, and each $S^{3}$ as a foliation of two circles, we have

$$
\begin{aligned}
& \mathrm{d} s_{10}^{2}=\mathrm{d} \varphi^{2}+ \lambda^{2}\left(\frac{1}{2}\left(-\cosh ^{2} \rho \mathrm{d} t^{2}+\mathrm{d} \rho^{2}+\sinh ^{2} \rho \mathrm{d} \gamma^{2}\right)+\left(\cos ^{2} \theta \mathrm{d} \psi^{2}+\mathrm{d} \theta^{2}+\sin ^{2} \theta \mathrm{d} \phi^{2}\right)\right. \\
&\left.+\left(\cos ^{2} \tilde{\theta} \mathrm{d} \tilde{\psi}^{2}+\mathrm{d} \tilde{\theta}^{2}+\sin ^{2} \tilde{\theta} \mathrm{d} \tilde{\phi}^{2}\right)\right) \\
& F_{(3)}=\lambda^{2}\left(\epsilon_{(3)}+2 \Omega_{(3)}+2 \widetilde{\Omega}_{(3)}\right)
\end{aligned}
$$


where $\epsilon_{(3)}, \Omega_{(3)}$ and $\widetilde{\Omega}_{(3)}$ are the volume-forms of the unit $\operatorname{AdS}_{(3)}$ and 3-spheres:

$\epsilon_{(3)}=\cosh \rho \sinh \rho \mathrm{d} t \wedge \mathrm{d} \rho \wedge \mathrm{d} \gamma, \quad \Omega_{(3)}=\cos \theta \sin \theta \mathrm{d} \psi \wedge \mathrm{d} \theta \wedge \mathrm{d} \phi$,

$\widetilde{\Omega}_{(3)}=\cos \tilde{\theta} \sin \tilde{\theta} \mathrm{d} \tilde{\psi} \wedge \mathrm{d} \tilde{\theta} \wedge \mathrm{d} \tilde{\phi}$.

After rotating the coordinates as follows,

$$
\psi=\cos \alpha \psi_{1}+\sin \alpha \psi_{2}, \quad \tilde{\psi}=-\sin \alpha \psi_{1}+\cos \alpha \psi_{2},
$$

the Penrose limit can be taken to be

$$
\begin{aligned}
& \rho \longrightarrow \frac{\rho}{\lambda}, \quad \theta \longrightarrow \frac{\theta}{\lambda}, \quad \tilde{\theta} \longrightarrow \frac{\tilde{\theta}}{\lambda}, \\
& t=x^{+}+\frac{x^{-}}{\lambda^{2}}, \quad \psi_{1}=\frac{1}{\sqrt{2}}\left(x^{+}-\frac{x^{-}}{\lambda^{2}}\right), \quad \psi_{2} \longrightarrow \frac{\psi_{2}}{\lambda},
\end{aligned}
$$

with the constant $\lambda$ set to infinity. By this means, the solution becomes the pp-wave

$$
\begin{aligned}
& \mathrm{d} s_{10}^{2}=-2 \mathrm{~d} x^{+} \mathrm{d} x^{-}+H \mathrm{~d} x^{+2}+\mathrm{d} z_{i}^{2}, \\
& F_{(3)}=\mathrm{d} x^{+} \wedge \Phi_{(2)},
\end{aligned}
$$

where $\Phi_{(2)}$ is a constant 2-form, given by

$$
\Phi_{(2)}=\left(2 \mathrm{~d} z_{1} \wedge \mathrm{d} z_{2}+\sqrt{2} \cos \alpha \mathrm{d} z_{3} \wedge \mathrm{d} z_{4}-\sqrt{2} \sin \alpha \mathrm{d} z_{5} \wedge \mathrm{d} z_{6}\right),
$$

and

$H=-\sum_{i=1}^{8} \mu_{i}^{2} z_{i}^{2} \equiv-\left(z_{1}^{2}+z_{2}^{2}\right)-\frac{1}{2} \cos ^{2} \alpha\left(z_{3}^{2}+z_{4}^{2}\right)-\frac{1}{2} \sin ^{2} \alpha\left(z_{5}^{2}+z_{6}^{2}\right)$.

The coordinates $z_{i}$ are defined to be

$z_{1}=\frac{1}{\sqrt{2}} \rho \cos \gamma, \quad z_{2}=\frac{1}{\sqrt{2}} \rho \sin \gamma, \quad z_{3}=\theta \cos \phi, \quad z_{4}=\theta \sin \phi$,

$z_{5}=\tilde{\theta} \cos \tilde{\phi}, \quad z_{6}=\tilde{\theta} \sin \tilde{\phi}, \quad z_{7}=\psi_{2}, \quad z_{8}=\varphi$.

Note that it is straightforward to introduce a parameter $\mu$ by scaling $x^{+} \rightarrow \mu x^{+}$and $x^{-} \rightarrow x^{-} / \mu$. In this paper we shall set $\mu=1$.

The above 5-5-1 system involves only the metric, the dilaton and the 3-form field strength, and is thus valid for the type IIA, type IIB and heterotic theories. It can be further lifted to $D=11$ as the 5-5-2 system. The near-horizon structure $\mathrm{AdS}_{3} \times S^{3} \times S^{3} \times S^{1}$ given in (4) can also be supported by a 4 -form field strength of the type IIA theory; it is given by

$$
F_{(4)}=\lambda^{2}\left(\epsilon_{(3)}+\Omega_{(3)}+\widetilde{\Omega}_{(3)}\right) \wedge \mathrm{d} \varphi .
$$

However, the brane interpretation becomes obscured in this case. The Penrose limit is now supported by the 4-form $F_{(4)}=\mathrm{d} x^{+} \wedge \Phi_{(2)} \wedge \mathrm{d} z_{8}$. Performing $T$-duality on the coordinate $\varphi$ leads to a solution supported by the R-R 3 -form in type IIB theory.

\section{Supersymmetry and string actions}

\subsection{Supersymmetry}

3.1.1. M-theory and type II viewpoints. A simple way of studying the supersymmetry of the pp-wave solution obtained in the previous section is to lift the solution to $D=11$. The resulting pp-wave is supported by the 4-form in $D=11$, given by

$$
F_{(4)}=\mathrm{d} x^{+} \wedge \Phi_{(2)} \wedge \mathrm{d} z_{9},
$$


where $z_{9}$ is the 11 th direction ${ }^{3}$. This 4 -form configuration is a special case of a general class of M-theory pp-waves considered in [23] where the supersymmetry of these solutions was discussed. It is straightforward to apply the general formalism of [23] to our solution. To do so, we introduce

$$
W=\frac{1}{2} \Phi_{i j} \Gamma^{i j 9}=2 \Gamma^{12}+\sqrt{2} \cos \alpha \Gamma^{34}-\sqrt{2} \sin \alpha \Gamma^{56}
$$

As discussed in [23], there are 16 standard Killing spinors associated with a generic pp-wave solution, which satisfy the projection

$$
\Gamma_{-} \epsilon=0 .
$$

In addition, since $\mu_{8,9}=0$, supernumerary Killing spinors arise if $W$ has zero eigenvalues, which occurs only for

$$
\cos ^{2} \alpha=\sin ^{2} \alpha=\frac{1}{2} \text {. }
$$

Using the formalism of [23] we find that, for the above $\alpha$, there are four supernumerary Killing spinors, all of which are independent of $x^{+}$and $z^{i}$ for $i=7,8,9$. The 16 standard Killing spinors are also independent of $z^{i}$ for $i=7,8,9$, but only 4 out of the 16 are independent of $x^{+}$. For other values of $\alpha$, there are no supernumerary Killing spinors, and all the standard Killing spinors depend on $x^{+}$. For this reason, we mainly focus on the case with $\alpha$ given by (17).

Since the Killing spinors are independent of $z_{7,8,9}$, we can perform dimensional reduction and $T$-duality on these directions without breaking any supersymmetry, even at the level of field theory. Thus, the above analysis of supersymmetry carries over to the type IIA and IIB theories.

3.1.2. Heterotic viewpoint. To study the number of Killing spinors of the solution in the heterotic theory, we can use the same Killing-spinor calculations as above. However, we must impose an additional ten-dimensional chirality condition on the Killing spinor:

$$
\epsilon=\Gamma_{9} \epsilon
$$

where $z_{9}$ is our 11 th coordinate. This has the effect of reducing the number of standard Killing spinors down to eight, two of which are independent of $x^{+}$. The number of supernumerary Killing spinors is now two. If we had chosen a different convention for the chirality projection, then there would not be any supernumerary Killing spinors at all. This implies that the sign choice of $F_{(3)}$ is important for the supernumerary Killing spinor in the heterotic theory.

\subsection{String action}

The pp-waves for which the function $H$ is quadratic in the transverse space coordinates are of particular interest because they provide backgrounds for string theory that are exactly solvable. The light-cone string action of the pp-wave arising from the Penrose limit of $\mathrm{AdS}_{5} \times S^{5}$ was studied in $[4,5]$. The action associated with the Penrose limit of the D1/D5 system can be found in [5, 6]. In [31], the Green-Schwarz action for type IIA and IIB strings in an arbitrary bosonic background was derived in component form up to second order in the fermionic coordinates. From these, type IIA and IIB string actions were obtained in the light-cone gauge on the background of a large class of pp-wave solutions [15, 23]. Applying the formalism

3 If we lift the pp-wave supported by the 4-form in $D=10, z_{9}$ is replaced by $z_{8}$, and hence these two solutions are totally equivalent in $D=11$. 
in $[15,23]$ to our example, one finds that the solution supported purely by $\mathrm{R}-\mathrm{R}$ fields is particularly simple. The bosonic string action is given by

$$
\mathcal{L}_{B}=-\frac{1}{2} \dot{z}^{2}-\frac{1}{2} z^{\prime 2}-\frac{1}{2} \mu_{i}^{2} z_{i}^{2}
$$

where the masses of the bosonic fields $\mu_{i}$ are defined in (11), given by

$$
\mu_{i}^{2}=\left\{1,1, \frac{1}{4} \frac{1}{4} \frac{1}{4} \frac{1}{4}, 0,0\right\} .
$$

For the solution supported by the type IIA 4-form field strength, the fermionic Lagrangian is given by

$$
\mathcal{L}_{F}=\bar{\Psi} \Gamma_{+}\left(\not \partial+\mu \varrho_{0} \Gamma_{8} W\right) \Psi,
$$

where $\varrho_{i}$, with $i=0,1,2$, are the worldsheet Dirac matrices, acting on the upper and lower 16 components of the column vector $\Psi$, and $\varrho_{2}$ is the chirality operator. The fermionic Lagrangian associated with the type IIB solution supported by the R-R 3-form has a similar structure, given by

$$
\mathcal{L}_{F}=\bar{\Psi} \Gamma_{+}\left(\not \partial+\mu \varrho_{2} W\right) \Psi .
$$

As discussed in $[5,15,23]$, the existence of $x^{+}$-independent supernumerary Killing spinors ensures that the corresponding string action has linearly-realized supersymmetry. In our case, the masses of the bosonic fields $z_{i}$ are given by (20). This precisely matches the masses of the fermonic fields, given by the eigenvalues of $W$. This is consistent with the supersymmetry. If we choose a different value of the rotation angle $\alpha$, two bosonic scalars remain massless, whilst all the fermions become massive, thereby breaking the supersymmetry. Indeed, for generic values of $\alpha$, there are no supernumerary Killing spinors.

For solutions supported by the NS-NS 3-form, the string action is slightly more complicated, since the NS-NS 3-form couples to the worldsheet as well. It is given by

$$
\mathcal{L}=\sum_{i=1}^{8}\left(\frac{1}{2} \dot{z}-\frac{1}{2} z^{\prime 2}+\frac{1}{2} \mu \Phi_{j i} z^{j} z_{i}^{\prime}-\frac{1}{2} \mu_{i}^{2} z_{i}^{2}\right)+\bar{\Psi}\left(\not \partial+\frac{1}{4} \varrho W\right) \Psi .
$$

\section{T-duality and $S^{1}$-wrapped D3-brane}

The pp-wave solution in $D=10$ has an eight-dimensional transverse space. In our case, the function $H$ is independent of the coordinates $z_{7,8}$. Thus, the 'natural' transverse space of our solution is six dimensional. We can perform $T$-duality and $S$-duality to relate our solution to a D3-brane whose transverse space has six dimensions.

First, we perform $T$-duality along the $\partial / \partial x^{+}$Killing direction and obtain an NS-NS string solution given by

$$
\begin{aligned}
& \mathrm{d} s_{10}^{2}=H^{-3 / 4}\left(-\mathrm{d} t^{2}+\left(\mathrm{d} x+\mathcal{A}_{(1)}\right)^{2}\right)+H^{1 / 4} \mathrm{~d} z_{i}^{2}, \\
& F_{(3)}^{\mathrm{NS}}=\mathrm{d}\left(H^{-1} \mathrm{~d} t \wedge\left(\mathrm{d} x+\mathcal{A}_{(1)}\right)\right)+\mu\left(\mathrm{d} x+\mathcal{A}_{(1)}\right) \wedge \Phi_{(2)}, \\
& \mathrm{d} \mathcal{A}_{(1)}=\Phi_{(2)}, \quad \phi=\frac{1}{2} \log H .
\end{aligned}
$$

Note that, in this solution, the worldsheet coordinate $x$ is fibred over the transverse space. This type of $S^{1}$-fibred string solution was first obtained in [32]. Next, we perform $S$-duality such that the string is supported by the R-R 3-form of type IIB theory. $T$-dualizing the $z_{7,8}$ directions yields an $S^{1}$-wrapped D3-brane given by

$$
\begin{aligned}
& \mathrm{d} s_{10}^{2}=H^{-1 / 2}\left(-\mathrm{d} t^{2}+\left(\mathrm{d} x_{1}+\mathcal{A}_{(1)}\right)^{2}+\mathrm{d} x_{2}^{2}+\mathrm{d} x_{3}^{2}\right)+H^{1 / 2} \mathrm{~d} z_{i}^{2}, \\
& F_{(5)}=\mathrm{d}\left(H^{-1} \mathrm{~d} t \wedge\left(\mathrm{d} x_{1}+\mathcal{A}_{(1)}\right) \wedge \mathrm{d} x_{2} \wedge \mathrm{d} x_{3}\right)+\mu\left(\mathrm{d} x_{1}+\mathcal{A}_{(1)}\right) \wedge \mathrm{d} x_{2} \wedge \mathrm{d} x_{3} \wedge \Phi_{(2)}+\text { dual, } \\
& \mathrm{d} \mathcal{A}_{(1)}=\Phi_{(2)} .
\end{aligned}
$$


An $S^{1}$-wrapped D3-brane was first constructed in [33] in order to resolve the singularity while maintaining the supersymmetry of the associated D3-brane on the resolved conifold. Here, when the D3-brane charge is turned on, the more general solution for $H$ is given by

$$
H=1+\frac{Q}{r^{4}}-\sum_{i=1}^{6} \mu_{i}^{2} z_{i}^{2},
$$

where $r^{2}=z^{i} z^{i}$. The terms associated with $\mu_{i}$ break the conformal symmetry. They introduce a naked singularity at some finite $r$, which can be argued [15] to be associated with a phase transition from type IIB theory to type IIB* theory, introduced in [34]. The solution has four Killing spinors for non-vanishing $Q$. For $Q=0$, or in the limit $r \rightarrow \infty$, four additional Killing spinors emerge.

\section{Further examples}

In this section, we consider further examples of non-standard intersections that give rise to AdS structure and study their Penrose limits. These examples can be generated from the above $\mathrm{AdS}_{3} \times S^{3} \times S^{3}$ by noting that $\mathrm{AdS}_{3}$ and $S^{3}$ can be (locally) expressed as a $U(1)$ bundle over $\mathrm{AdS}_{2}$ and $S^{2}$ respectively, i.e.,

$$
\begin{array}{ll}
\mathrm{d} s_{\mathrm{AdS}_{3}}^{2}=\frac{1}{4}\left(\mathrm{~d} z+\mathcal{A}_{(1)}\right)^{2}+\frac{1}{4} \mathrm{~d} s_{\mathrm{AdS}_{2},}^{2} & \mathrm{~d} \mathcal{A}_{(1)}=\epsilon_{(2)}, \\
\mathrm{d} \Omega_{3}^{2}=\frac{1}{4}\left(\mathrm{~d} z+\mathcal{B}_{(1)}\right)^{2}+\frac{1}{4} \mathrm{~d} \Omega_{2}^{2}, & \mathrm{~d} \mathcal{B}_{(1)}=\Omega_{(2)},
\end{array}
$$

where $\epsilon_{(2)}$ and $\Omega_{(2)}$ are the volume-forms of the unit $\mathrm{AdS}_{2}$ and $S^{2}$, respectively. The bundle can be naturally generated by considering a wave in $\mathrm{AdS}_{3}$ or a Taub-NUT in $R^{4}$. From the $D=10$ perspective, the wave and NUT in eleven dimensions become a D0-brane and D6-brane, respectively. The intersecting systems of this section can be found in [35].

\subsection{Type IIA examples}

D0/D4/D4/NS1 system. The solution for the D0/D4/D4/NS1 system is given by [35]

$$
\begin{aligned}
& \mathrm{d} s_{10}^{2}=H_{0}^{-7 / 8} H_{4}^{-3 / 8} \widetilde{H}_{4}^{-3 / 8} H_{1}^{-3 / 4}\left(-\mathrm{d} t^{2}+H_{0} H_{4} \widetilde{H}_{4} \mathrm{~d} x^{2}+H_{0} H_{4} H_{1} \mathrm{~d} y_{i}^{2}+H_{0} \widetilde{H}_{4} H_{1} \mathrm{~d} \widetilde{y}_{i}^{2}\right), \\
& F_{(4)}=\mathrm{e}^{-\frac{1}{2} \phi} *\left(\widetilde{H}_{4} \mathrm{~d} t \wedge \mathrm{d}^{4} \tilde{y} \wedge \mathrm{d} H_{4}^{-1}\right)+\mathrm{e}^{-\frac{1}{2} \phi} *\left(H_{4} \mathrm{~d} t \wedge \mathrm{d}^{4} y \wedge \mathrm{d} \widetilde{H}_{4}^{-1}\right), \\
& F_{(3)}=\mathrm{d} t \wedge \mathrm{d} x \wedge \mathrm{d} H_{1}^{-1}, \quad F_{(2)}=\mathrm{d} t \wedge \mathrm{d} H_{0}^{-1}, \\
& \phi=-\frac{1}{4} \log \left[H_{4} \widetilde{H}_{4} H_{1}^{2} / H_{0}^{3}\right], \quad H_{0}=H_{1}=H_{4} \widetilde{H}_{4},
\end{aligned}
$$

where $H_{4}=H_{4}\left(y_{i}\right)$ and $\widetilde{H}_{4}=\widetilde{H}_{4}\left(\widetilde{y}_{i}\right)$. The solution can be represented diagrammatically as follows:

\begin{tabular}{c|ccccccccccc} 
& $t$ & $x$ & $y_{1}$ & $y_{2}$ & $y_{3}$ & $y_{4}$ & $\tilde{y}_{1}$ & $\tilde{y}_{2}$ & $\tilde{y}_{3}$ & $\tilde{y}_{4}$ & \\
\hline D0 & $\times$ & - & - & - & - & - & - & - & - & - & $H_{0}$ \\
D4 & $\times$ & - & $\times$ & $\times$ & $\times$ & $\times$ & - & - & - & - & $\tilde{H}_{4}$ \\
D4 & $\times$ & - & - & - & - & - & $\times$ & $\times$ & $\times$ & $\times$ & $H_{4}$ \\
NS1 & $\times$ & $\times$ & - & - & - & - & - & - & - & - & $H_{1}$
\end{tabular}


We parametrize the coordinates as $\mathrm{d} y_{i}^{2}=\mathrm{d} y^{2}+y^{2} \mathrm{~d} \Omega_{3}^{2}$ and $\mathrm{d} \widetilde{y}_{i}^{2}=\mathrm{d} \widetilde{y}^{2}+\widetilde{y}^{2} \mathrm{~d} \widetilde{\Omega}_{3}^{2}$, so that $H_{4}$ and $\widetilde{H}_{4}$ are given by (3). For simplicity, we have equal charges. The near-horizon geometry is $\mathrm{AdS}_{2} \times S^{3} \times S^{3} \times T^{2}$ [35], namely

$\mathrm{d} s_{10}^{2}=\lambda^{2}\left(\frac{1}{8} \mathrm{~d} s_{\mathrm{AdS}}^{2}+\mathrm{d} \Omega_{3}^{2}+\mathrm{d} \widetilde{\Omega}_{3}^{2}\right)+\mathrm{d} \varphi_{1}^{2}+\mathrm{d} \varphi_{2}^{2}$,

$F_{(4)}=2 \lambda^{2}\left(\Omega_{(3)}+\widetilde{\Omega}_{3}\right) \wedge \mathrm{d} \varphi_{1}, \quad F_{(3)}=\frac{\lambda}{\sqrt{8}} \epsilon_{(2)} \wedge \mathrm{d} \varphi_{1}, \quad F_{(2)}=\frac{\lambda}{\sqrt{8}} \epsilon_{(2)}$.

Performing the Penrose limit, we have

$$
\begin{aligned}
& \mathrm{d} s_{10}^{2}=-2 \mathrm{~d} x^{+} \mathrm{d} x^{-}+H \mathrm{~d} x^{+2}+\mathrm{d} z_{i}^{2}, \\
& F_{(4)}=\mathrm{d} x^{+} \wedge \Phi_{(3)}, \quad F_{(3)}=\mathrm{d} x^{+} \wedge \Phi_{(2)}, \quad F_{(2)}=\mathrm{d} x^{+} \wedge \Phi_{(1)},
\end{aligned}
$$

where the constant form fields are given by

$$
\begin{aligned}
& \Phi_{(3)}=\frac{1}{\sqrt{2}}\left(\cos \alpha \mathrm{d} z_{2} \wedge \mathrm{d} z_{3}-\sin \alpha \mathrm{d} z_{4} \wedge \mathrm{d} z_{5}\right) \wedge \mathrm{d} z_{7}, \\
& \Phi_{(2)}=\mathrm{d} z_{7} \wedge \mathrm{d} z_{1}, \quad \Phi_{(1)}=\mathrm{d} z_{1},
\end{aligned}
$$

and

$$
H=-z_{1}^{2}-\frac{1}{8} \cos ^{2} \alpha\left(z_{2}^{2}+z_{3}^{2}\right)-\frac{1}{8} \sin ^{2} \alpha\left(z_{4}^{2}+z_{5}^{2}\right) .
$$

It is worth observing that in this pp-wave solution, all the form fields of the type IIA theory are turned on.

Note that we can $T$-dualize along the $\partial / \partial x^{+}$Killing direction and obtain a deformed $S^{1}$-wrapped type IIB NS string solution. Then, by $T$-dualizing along $z_{8}$ and lifting to $D=11$, one obtains a deformed $T^{2}$-wrapped M2-brane solution of M-theory given by

$\mathrm{d} s_{11}^{2}=H^{-2 / 3}\left(-\mathrm{d} t^{2}+\left(\mathrm{d} x_{1}+\mathcal{A}_{(1)}\right)^{2}+\left(\mathrm{d} x_{2}+\mathcal{B}_{(1)}\right)^{2}\right)+H^{1 / 3} \mathrm{~d} z_{i}^{2}$,

$F_{(4)}=\mathrm{d}\left(H^{-1} \mathrm{~d} t \wedge\left(\mathrm{d} x_{1}+\mathcal{A}_{(1)}\right) \wedge\left(\mathrm{d} x_{2}+\mathcal{B}_{(1)}\right)\right)+\left[\Phi_{(3)} \wedge \mathrm{d} z_{8}+8\right.$-dim dual $]$,

$\mathrm{d} \mathcal{A}_{(1)}=\Phi_{(2)}, \quad \mathrm{d} \mathcal{B}_{(1)}=\Phi_{(1)} \wedge \mathrm{d} z_{8}$,

D2/D4/D6/NS5 system. The D2/D4/D6/NS5 solution is given by [35]

$\mathrm{d} s_{10}^{2}=H_{2}^{-5 / 8} H_{4}^{-3 / 8} H_{6}^{-1 / 8} H_{5}^{-1 / 4}\left(-\mathrm{d} t^{2}+\mathrm{d} x_{1}^{2}+H_{4} H_{5} \mathrm{~d} x_{2}^{2}+H_{2} H_{6} H_{5} \mathrm{~d} y_{i}^{2}+H_{2} H_{4} \mathrm{~d} z_{j}^{2}\right)$,

$F_{(4)}=\mathrm{d} t \wedge \mathrm{d} x_{1} \wedge \mathrm{d} x_{2} \wedge \mathrm{d} H_{2}^{-1}+\mathrm{e}^{-\frac{1}{2} \phi} *\left(\mathrm{~d} t \wedge \mathrm{d} x_{1} \wedge \mathrm{d}^{3} y \wedge \mathrm{d} H_{4}^{-1}\right)$,

$F_{(3)}=\mathrm{e}^{\phi} *\left(\mathrm{~d} t \wedge \mathrm{d} x_{1} \wedge \mathrm{d}^{4} z \wedge \mathrm{d} H_{5}^{-1}\right), \quad F_{(2)}=\mathrm{e}^{-\frac{3}{2} \phi} *\left(\mathrm{~d} t \wedge \mathrm{d} x_{1} \wedge \mathrm{d} x_{2} \wedge \mathrm{d}^{4} z \wedge \mathrm{d} H_{6}^{-1}\right)$,

$\phi=\frac{1}{4} \log \left[H_{2} H_{5}^{2} /\left(H_{4} H_{6}^{3}\right)\right], \quad H_{2}=H_{4}\left(z_{j}\right) H\left(y_{i}\right)$,

where $H_{4}=H_{4}\left(z_{j}\right), H_{6}=H_{5}=H\left(y_{i}\right)$. The solution can be represented diagrammatically as follows:

\begin{tabular}{c|ccccccccccc} 
& $t$ & $x_{1}$ & $x_{2}$ & $y_{1}$ & $y_{2}$ & $y_{3}$ & $z_{1}$ & $z_{2}$ & $z_{3}$ & $z_{4}$ & \\
\hline D2 & $\times$ & $\times$ & $\times$ & - & - & - & - & - & - & - & $H_{2}$ \\
D4 & $\times$ & $\times$ & - & $\times$ & $\times$ & $\times$ & - & - & - & - & $H_{4}$ \\
D6 & $\times$ & $\times$ & $\times$ & - & - & - & $\times$ & $\times$ & $\times$ & $\times$ & $H_{6}$ \\
NS5 & $\times$ & $\times$ & - & - & - & - & $\times$ & $\times$ & $\times$ & $\times$ & $H_{5}$
\end{tabular}

The D2/D4/D6/NS5 system

The near-horizon geometry is $\mathrm{AdS}_{3} \times S^{3} \times S^{2} \times T^{2}$ [35], given by $\mathrm{d} s_{10}^{2}=\lambda^{2}\left(\frac{1}{2} \mathrm{~d} S_{\mathrm{AdS}_{3}}^{2}+\mathrm{d} \Omega_{3}^{2}+\frac{1}{4} \mathrm{~d} \widetilde{\Omega}_{2}^{2}\right)+\mathrm{d} \varphi_{1}^{2}+\mathrm{d} \varphi_{2}^{2}$,

$F_{(4)}=\lambda^{2}\left(\epsilon_{(3)}+2 \Omega_{(3)}\right) \wedge \mathrm{d} \varphi_{1}, \quad F_{(3)}=\frac{1}{2} \lambda \widetilde{\Omega}_{(2)} \wedge \mathrm{d} \varphi_{1}, \quad F_{(2)}=\frac{1}{2} \lambda \widetilde{\Omega}_{(2)}$. 
The Penrose limit is given by

$$
\begin{aligned}
& \mathrm{d} s_{10}^{2}=-2 \mathrm{~d} x^{+} \mathrm{d} x^{-}+H \mathrm{~d} x^{+2}+\mathrm{d} z_{i}^{2}, \\
& F_{(4)}=\mathrm{d} x^{+} \wedge \Phi_{(3)}, \quad F_{(3)}=\mathrm{d} x^{+} \wedge \Phi_{(2)}, \quad F_{(2)}=\mathrm{d} x^{+} \wedge \Phi_{(1)},
\end{aligned}
$$

where the constant form fields are given by

$$
\begin{aligned}
& \Phi_{(3)}=\left(2 \mathrm{~d} z_{1} \wedge \mathrm{d} z_{2}+\sqrt{2} \cos \alpha \mathrm{d} z_{3} \wedge \mathrm{d} z_{4}\right) \wedge \mathrm{d} z_{6}, \\
& \Phi_{(2)}=\sqrt{2} \sin \alpha \mathrm{d} z_{5} \wedge \mathrm{d} z_{6}, \quad \Phi_{(1)}=\sqrt{2} \sin \alpha \mathrm{d} z_{5},
\end{aligned}
$$

and

$$
H=-\left(z_{1}^{2}+z_{2}^{2}\right)-\frac{1}{2} \cos ^{2} \alpha\left(z_{3}^{2}+z_{4}^{2}\right)-2 \sin ^{2} \alpha z_{5}^{2} .
$$

As in the previous example, by $T$-duality this solution is related to a deformed $T^{2}$-wrapped M2-brane solution of M-theory.

D2/D4/D4/NS1/NS5/NS5 system. The maximal number of intersecting components that give rise to an AdS structure is six, namely the D2/D4/D4/NS1/NS5/NS5 solution, which is given by [35]

$$
\begin{aligned}
& \mathrm{d} s_{10}^{2}=H_{2}^{-5 / 8} H_{4}^{-3 / 8} \widetilde{H}_{4}^{-3 / 8} H_{1}^{-3 / 4} H_{5}^{-1 / 4} \widetilde{H}_{5}^{-1 / 4}\left(-\mathrm{d} t^{2}+H_{2} H_{4} \widetilde{H}_{4} \mathrm{~d} x_{1}^{2}+H_{4} H_{1} \widetilde{H}_{5} \mathrm{~d} x_{2}^{2}\right. \\
& \left.+\widetilde{H}_{4} H_{1} H_{5} \mathrm{~d} x_{3}^{2}+H_{2} H_{4} H_{1} H_{5} \mathrm{~d} y_{i}^{2}+H_{2} \widetilde{H}_{4} H_{1} \widetilde{H}_{5} \mathrm{~d} \widetilde{y}_{i}^{2}\right), \\
& F_{(4)}=\mathrm{d} t \wedge \mathrm{d} x_{2} \wedge \mathrm{d} x_{3} \wedge \mathrm{d} H_{2}^{-1}+\mathrm{e}^{-\frac{1}{2} \phi} *\left(\widetilde{H}_{4} \mathrm{~d} t \wedge \mathrm{d} x_{3} \wedge \mathrm{d}^{3} \tilde{y} \wedge \mathrm{d} H_{4}^{-1}\right) \\
& +\mathrm{e}^{-\frac{1}{2} \phi} *\left(H_{4} \mathrm{~d} t \wedge \mathrm{d} x_{2} \wedge \mathrm{d}^{3} y \wedge \mathrm{d} \widetilde{H}_{4}^{-1}\right), \\
& F_{(3)}=\mathrm{d} t \wedge \mathrm{d} x_{1} \wedge \mathrm{d} H_{1}^{-1}+\mathrm{e}^{\phi} *\left(\widetilde{H}_{5} \mathrm{~d} t \wedge \mathrm{d} x_{1} \wedge \mathrm{d} x_{2} \wedge \mathrm{d}^{3} \widetilde{y} \wedge \mathrm{d} H_{5}^{-1}\right) \\
& +\mathrm{e}^{\phi} *\left(H_{5} \mathrm{~d} t \wedge \mathrm{d} x_{1} \wedge \mathrm{d} x_{3} \wedge \mathrm{d}^{3} y \wedge \mathrm{d} \widetilde{H}_{5}^{-1}\right), \\
& \phi=\frac{1}{4} \log \left[H_{2}\left(H_{5} \widetilde{H}_{5}\right)^{2} /\left(H_{1}^{2} H_{4} \widetilde{H}_{4}\right)\right], \quad H_{2}=H_{1}=H\left(y_{i}\right) \tilde{H}\left(\tilde{y}_{i}\right),
\end{aligned}
$$

where $H_{4}=H_{5}=H\left(y_{i}\right)$ and $\widetilde{H}_{4}=\widetilde{H}_{5}=\widetilde{H}\left(\widetilde{y}_{i}\right)$. The solution can be represented diagrammatically as follows:

\begin{tabular}{c|ccccccccccc} 
& $t$ & $x_{1}$ & $x_{2}$ & $x_{3}$ & $y_{1}$ & $y_{2}$ & $y_{3}$ & $\tilde{y}_{1}$ & $\tilde{y}_{2}$ & $\tilde{y}_{3}$ & \\
\hline D2 & $\times$ & - & $\times$ & $\times$ & - & - & - & - & - & - & $H_{2}$ \\
D4 & $\times$ & - & - & $\times$ & - & - & - & $\times$ & $\times$ & $\times$ & $H_{4}$ \\
D4 & $\times$ & - & $\times$ & - & $\times$ & $\times$ & $\times$ & - & - & - & $\tilde{H}_{4}$ \\
NS1 & $\times$ & $\times$ & - & - & - & - & - & - & - & - & $H_{1}$ \\
NS5 & $\times$ & $\times$ & $\times$ & - & - & - & - & $\times$ & $\times$ & $\times$ & $H_{5}$ \\
NS5 & $\times$ & $\times$ & - & $\times$ & $\times$ & $\times$ & $\times$ & - & - & - & $\tilde{H}_{5}$
\end{tabular}

D2/D4/D4/NS1/NS5/NS5 system

We parametrize the coordinates as $\mathrm{d} y_{i}^{2}=\mathrm{d} y^{2}+y^{2} \mathrm{~d} \Omega_{2}^{2}$ and $\mathrm{d} \widetilde{y}_{i}^{2}=\mathrm{d} \widetilde{y}^{2}+\widetilde{y}^{2} \mathrm{~d} \widetilde{\Omega}_{2}^{2}$, so that

$$
H_{4}=H_{5}=1+\frac{\lambda}{y}
$$

and likewise for $\widetilde{H}_{4}$ and $\widetilde{H}_{5}$. The near-horizon geometry is $\operatorname{AdS}_{2} \times S^{2} \times S^{2} \times T^{4}$ [35]. Explicitly, we have

$$
\begin{aligned}
& \mathrm{d} s_{10}^{2}=\frac{1}{4} \lambda^{2}\left(\frac{1}{2} \mathrm{~d} s_{\mathrm{AdS}_{2}}^{2}+\mathrm{d} \Omega_{(2)}^{2}+\mathrm{d} \widetilde{\Omega}_{2}^{2}\right)+\mathrm{d} \varphi_{1}^{2}+\mathrm{d} \varphi_{2}^{2}+\mathrm{d} \varphi_{3}^{2}+\mathrm{d} \varphi_{4}^{2}, \\
& F_{(4)}=\frac{1}{2} \lambda\left(\Omega_{(2)} \wedge \mathrm{d} \varphi_{1} \wedge \mathrm{d} \varphi_{3}+\widetilde{\Omega}_{(2)} \wedge \mathrm{d} \varphi_{1} \wedge \mathrm{d} \varphi_{4}+\frac{1}{\sqrt{2}} \epsilon_{(2)} \wedge \mathrm{d} \varphi_{3} \wedge \mathrm{d} \varphi_{4}\right), \\
& F_{(3)}=\frac{1}{2} \lambda\left(\Omega_{(2)} \wedge \mathrm{d} \varphi_{4}+\widetilde{\Omega}_{2} \wedge \mathrm{d} \varphi_{3}+\frac{1}{\sqrt{2}} \epsilon_{(2)} \wedge \mathrm{d} \varphi_{4}\right) .
\end{aligned}
$$


We find that the Penrose limit of this system is given by

$$
\begin{aligned}
& \mathrm{d} s_{10}^{2}=-2 \mathrm{~d} x^{+} \mathrm{d} x^{-}+H \mathrm{~d} x^{+2}+\mathrm{d} z_{i}^{2}, \\
& F_{(4)}=\mathrm{d} x^{+} \wedge \Phi_{(3)}, \quad F_{(3)}=\mathrm{d} x^{+} \wedge \Phi_{(2)},
\end{aligned}
$$

where the constant form fields are given by

$\Phi_{(3)}=\sqrt{2}\left(\mathrm{~d} z_{6} \wedge \mathrm{d} z_{7} \wedge \mathrm{d} z_{1}+\frac{1}{2} \cos \alpha \mathrm{d} z_{2} \wedge \mathrm{d} z_{5} \wedge \mathrm{d} z_{6}-\frac{1}{2} \sin \alpha \mathrm{d} z_{3} \wedge \mathrm{d} z_{5} \wedge \mathrm{d} z_{7}\right)$,

$\Phi_{(2)}=\sqrt{2}\left(\mathrm{~d} z_{5} \wedge \mathrm{d} z_{1}+\frac{1}{2} \cos \alpha \mathrm{d} z_{2} \wedge \mathrm{d} z_{7}-\frac{1}{2} \sin \alpha \mathrm{d} z_{3} \wedge \mathrm{d} z_{6}\right)$,

and

$$
H=-z_{1}^{2}-\frac{1}{2} \cos ^{2} \alpha z_{2}^{2}-\frac{1}{2} \sin ^{2} \alpha z_{3}^{2} .
$$

Note that this solution can be easily lifted to $D=11$ as the Penrose limit of the M2/M2/M5/M5/M5/M5 system. There are six terms in the 4-form field stength in the eleven-dimensional pp-wave solution. This is a special case of the general class of M-theory pp-wave solutions obtained in [23].

\subsection{Type IIB examples}

D3/D1/D5/NS1/NS5 system. The near-horizon geometry of this system is $\operatorname{AdS}_{2} \times S^{3} \times$ $S^{2} \times T^{3}$ [35]. Explicitly, we find that

$$
\begin{aligned}
& \mathrm{d} s_{10}^{2}=\lambda^{2}\left(\frac{1}{8} \mathrm{~d} s_{\mathrm{AdS} S_{2}}^{2}+\mathrm{d} \Omega_{3}^{2}+\frac{1}{4} \mathrm{~d} \widetilde{\Omega}_{2}^{2}\right)+\mathrm{d} \varphi_{1}^{2}+\mathrm{d} \varphi_{2}^{2}+\mathrm{d} \varphi_{3}^{2}, \\
& F_{(5)}=2 \lambda^{2} \Omega_{(3)} \wedge \mathrm{d} \varphi_{1} \wedge \mathrm{d} \varphi_{3}+\text { dual }, \\
& F_{(3)}^{\mathrm{RR}}=\frac{1}{2} \lambda\left(\widetilde{\Omega}_{(2)} \wedge \mathrm{d} \varphi_{1}+\frac{1}{\sqrt{2}} \epsilon_{(2)} \wedge \mathrm{d} \varphi_{3}\right), \\
& F_{(3)}^{\mathrm{NS}}=\frac{1}{2} \lambda\left(\widetilde{\Omega}_{(2)} \wedge \mathrm{d} \varphi_{3}+\frac{1}{\sqrt{2}} \epsilon_{(2)} \wedge \mathrm{d} \varphi_{1}\right) .
\end{aligned}
$$

The Penrose limit is given by

$$
\begin{aligned}
& \mathrm{d} s_{10}^{2}=-2 \mathrm{~d} x^{+} \mathrm{d} x^{-}+H \mathrm{~d} x^{+2}+\mathrm{d} z_{i}^{2}, \\
& F_{(5)}=\mathrm{d} x^{+} \wedge \Phi_{(4)}, \quad F_{(3)}^{\mathrm{RR}}=\mathrm{d} x^{+} \wedge \Phi_{(2)}^{\mathrm{RR}}, \quad F_{(3)}^{\mathrm{NS}}=\mathrm{d} x^{+} \wedge \Phi_{(2)}^{\mathrm{NS}},
\end{aligned}
$$

where the constant form fields are given by

$$
\begin{aligned}
& \Phi_{(4)}=\frac{1}{\sqrt{2}} \cos \alpha \mathrm{d} z_{2} \wedge \mathrm{d} z_{3} \wedge \mathrm{d} z_{6} \wedge \mathrm{d} z_{8}, \\
& \Phi_{(2)}^{\mathrm{RR}}=\mathrm{d} z_{1} \wedge \mathrm{d} z_{8}-\frac{1}{\sqrt{2}} \sin \alpha \mathrm{d} z_{4} \wedge \mathrm{d} z_{6}, \\
& \Phi_{(2)}^{\mathrm{NS}}=\mathrm{d} z_{1} \wedge \mathrm{d} z_{6}-\frac{1}{\sqrt{2}} \sin \alpha \mathrm{d} z_{4} \wedge \mathrm{d} z_{8},
\end{aligned}
$$

and

$$
H=-z_{1}^{2}-\frac{1}{8} \cos ^{2} \alpha\left(z_{2}^{2}+z_{3}^{2}\right)-\frac{1}{2} \sin ^{2} \alpha z_{4}^{2}
$$

D3/D5/D5/NS5/NS5 system. The near-horizon geometry of this system is $\mathrm{AdS}_{3} \times S^{2} \times$ $S^{2} \times T^{3}$ [35]. Explicitly, we have

$$
\begin{aligned}
& \mathrm{d} s_{10}^{2}=\lambda^{2}\left(\frac{1}{2} \mathrm{~d} s_{\mathrm{AdS}_{3}}^{2}+\frac{1}{4} \mathrm{~d} \Omega_{2}^{2}+\frac{1}{4} \mathrm{~d} \widetilde{\Omega}_{2}^{2}\right)+\mathrm{d} \varphi_{1}^{2}+\mathrm{d} \varphi_{2}^{2}+\mathrm{d} \varphi_{3}^{2}, \\
& F_{(5)}=\lambda^{2} \epsilon_{(3)} \wedge \mathrm{d} \varphi_{1} \wedge \varphi_{3}+\text { dual }, \\
& F_{(3)}^{\mathrm{RR}}=\frac{1}{2} \lambda\left(\Omega_{(2)} \wedge \mathrm{d} \varphi_{1}+\widetilde{\Omega}_{(2)} \wedge \mathrm{d} \varphi_{3}\right), \\
& F_{(3)}^{\mathrm{NS}}=\frac{1}{2} \lambda\left(\Omega_{(2)} \wedge \mathrm{d} \varphi_{3}+\widetilde{\Omega}_{(2)} \wedge \mathrm{d} \varphi_{1}\right),
\end{aligned}
$$


The Penrose limit is given by

$$
\begin{aligned}
& \mathrm{d} s_{10}^{2}=-2 \mathrm{~d} x^{+} \mathrm{d} x^{-}+H \mathrm{~d} x^{+2}+\mathrm{d} z_{i}^{2}, \\
& F_{(5)}=\mathrm{d} x^{+} \wedge \Phi_{(4)}, \quad F_{(3)}^{\mathrm{RR}}=\mathrm{d} x^{+} \wedge \Phi_{(2)}^{\mathrm{RR}}, \quad F_{(3)}^{\mathrm{NS}}=\mathrm{d} x^{+} \wedge \Phi_{(2)}^{\mathrm{NS}},
\end{aligned}
$$

where the constant form fields are given by

$$
\begin{aligned}
& \Phi_{(4)}=2 \mathrm{~d} z_{1} \wedge \mathrm{d} z_{2} \wedge \mathrm{d} z_{6} \wedge \mathrm{d} z_{8}, \\
& \Phi_{(2)}^{\mathrm{RR}}=\sqrt{2}\left(\cos \alpha \mathrm{d} z_{3} \wedge \mathrm{d} z_{6}-\sin \alpha \mathrm{d} z_{4} \wedge \mathrm{d} z_{8}\right), \\
& \Phi_{(2)}^{\mathrm{NS}}=\sqrt{2}\left(\cos \alpha \mathrm{d} z_{3} \wedge \mathrm{d} z_{8}-\sin \alpha \mathrm{d} z_{4} \wedge \mathrm{d} z_{6}\right),
\end{aligned}
$$

and

$$
H=-\left(z_{1}^{2}+z_{2}^{2}\right)-2 \cos ^{2} \alpha z_{3}^{2}-2 \sin ^{2} \alpha z_{4}^{2}
$$

\section{Conclusions}

We have considered the Penrose limits of $\mathrm{AdS}_{3}$ and $\mathrm{AdS}_{2}$ spacetimes that arise from various non-standard brane intersections in type IIA, IIB and heterotic theories. We have focused on the non-standard intersection of two 5-branes and one string, whose near-horizon geometry is $\mathrm{AdS}_{3} \times S^{3} \times S^{3} \times S^{1}$. This provides a new exact solvable string background for studying $\mathrm{AdS}_{3} / \mathrm{CFT}_{2}$ correspondence. A new feature is that the product spacetime involves two 3 -spheres, implying the isometry group is $S O(4) \times S O(4)$. After making an orthogonal rotation of the two foliating circles of the two 3-spheres, we obtain a one-parameter family of Penrose limits. Through the study of the supersymmetry of the resulting pp-wave, we find that supernumerary Killing spinors arise only for a $45^{\circ}$ rotation. Thus, there are four supernumerary Killing spinors in addition to the 16 standard Killing spinors. The existence of the supernumerary Killing spinors ensures that the corresponding light-cone string action has linearly-realized supersymmetry. It is of interest to further investigate the role of the variable rotation parameter for supersymmetry breaking.

\section{Acknowledgments}

We would like to thank Mirjam Cvetič and Chris Pope for useful conversations. HL was supported by DOE grant DE-FG02-95ER40899. JFV was supported by the Francqui Foundation (Belgium), the Actions de Recherche Concertées of the Direction de la Recherche Scientifique-Communauté Francaise de Belgique, IISN-Belgium (convention 4.4505.86).

\section{References}

[1] Kowalski-Glikman J 1984 Vacuum states in supersymmetric Kaluza-Klein theory Phys. Lett. B 134194

[2] Blau M, Figueroa-O'Farrill J, Hull C and Papadopoulos G 2002 A new maximally supersymmetric background of IIB superstring theory J. High Energy Phys. JHEP01(2002)047 (Preprint hep-th/0110242)

[3] Blau M, Figueroa-O'Farrill J, Hull C and Papadopoulos G 2002 Penrose limits and maximal supersymmetry Preprint hep-th/0201081

[4] Metsaev R R 2002 Type IIB Green-Schwarz superstring in plane wave Ramond-Ramond background Nucl. Phys. B 62570 (Preprint hep-th/0112044)

[5] Berenstein D, Maldacena J and Nastase H 2002 Strings in flat space and pp waves from $N=4$ super Yang-Mills Preprint hep-th/0202021

[6] Metsaev R R and Tseytlin A A 2002 Exactly solvable model of superstring in plane wave Ramond-Ramond background Preprint hep-th/0202109 
[7] Blau M, Figueroa-O'Farrill J and Papadopoulos G 2002 Penrose limits, supergravity and brane dynamics Preprint hep-th/0202111

[8] Itzhaki N, Klebanov I R and Mukhi S 2002 PP wave limit and enhanced supersymmetry in gauge theories Preprint hep-th/0202153

[9] Gomis J and Ooguri H 2002 Penrose limit of $N=1$ gauge theories Preprint hep-th/0202157

[10] Russo J G and Tseytlin A A 2002 On solvable models of type IIB superstring in NS-NS and R-R plane wave backgrounds Preprint hep-th/0202179

[11] Pando-Zayas L A and Sonnenschein J 2002 On Penrose limits and gauge theories Preprint hep-th/0202186

[12] Alishahiha M and Sheikh-Jabbari M M 2002 The pp-wave limits of orbifolded AdS $_{5} \times S^{5}$ Preprint hep-th/0203018

[13] Billo' M and Pesando I 2002 Boundary states for GS superstrings in an Hpp wave background Preprint hep-th/0203028

[14] Kim N, Pankiewicz A, Rey S-J and Theisen S 2002 Superstring on pp-wave orbifold from large- $N$ quiver gauge theory Preprint hep-th $/ 0203080$

[15] Cvetič M, Lü H and Pope C N 2002 Penrose limits, pp-waves and deformed M2-branes Preprint hep-th/0203082

[16] Takayanagi T and Terashima S 2002 Strings on orbifolded pp-waves Preprint hep-th/0203093

[17] Gursoy U, Nunez C and Schvellinger M 2002 RG flows from Spin(7), CY 4-fold and HK manifolds to AdS, Penrose limits and pp waves Preprint hep-th/0203124

[18] Floratos E and Kehagias A 2002 Penrose limits of orbifolds and orientifolds Preprint hep-th/0203134

[19] Michelson J 2002 (Twisted) toroidal compactification of pp-waves Preprint hep-th/0203140

[20] Gueven R 2002 Randall-Sundrum zero mode as a Penrose limit Preprint hep-th/0203153

[21] Das S R, Gomez C and Rey S J 2002 Penrose limit, spontaneous symmetry breaking and holography in pp-wave background Preprint hep-th/0203164

[22] Chu C S and Ho P M 2002 Noncommutative D-brane and open string in pp-wave background with B-field Preprint hep-th/0203186

[23] Cvetič M, Lü H and Pope C N 2002 M-theory pp-waves, Penrose limits and supernumerary supersymmetries Preprint hep-th/0203229

[24] Berenstein D, Gava E, Maldacena J, Narain K S and Nastase H 2002 Open strings on plane waves and their Yang-Mills duals Preprint hep-th/0203249

[25] Gauntlett J P and Hull C M 2002 PP-waves in 11-dimensions with extra supersymmetry Preprint hep-th/0203255

[26] Lee P and Park J W 2002 Open strings in PP-wave background from defect conformal field theory Preprint hep-th/0203257

[27] Khuri R R 1993 A comment on the string solitons Phys. Rev. D 482947 Preprint hep-th/9305143

[28] Behrndt K, Bergshoeff E and Janssen B 1997 Intersecting D-branes in ten and six dimensions Phys. Rev. D 55 3785 (Preprint hep-th/9604168)

[29] Gauntlett J P, Kastor D A and Traschen J 1996 Overlapping branes in M-theory Nucl. Phys. B 478544 (Preprint hep-th/9604179)

[30] Cowdall P M and Townsend P K 1998 Gauged supergravity vacua from intersecting branes Phys. Lett. B 429 281

Cowdall P M and Townsend P K 1998 Phys. Lett. B 434458 (erratum) (Preprint hep-th/9801165)

[31] Cvetič M, Lü H, Pope C N and Stelle K S 2000 T-duality in the Green-Schwarz formalism, and the massless/massive IIA duality map Nucl. Phys. B 573149 Preprint hep-th/9907202

[32] Lü H and Vázquez-Poritz J F 2002 Resolution of overlapping branes Preprint hep-th/0202075

[33] Lü H and Vázquez-Poritz J F $2002 \mathrm{~S}^{1}$-wrapped D3-branes on conifolds Preprint hep-th/0202175

[34] Hull C M 1998 Timelike T-duality, de Sitter space, large $N$ gauge theories and topological field theory J. High Energy Phys. JHEP07(1998)021 Preprint hep-th/9806146

[35] Boonstra H J, Peeters B and Skenderis K 1998 Brane intersections, anti-de Sitter spacetimes and dual superconformal field theories Nucl. Phys. B 533 127-62 (Preprint hep-th/9803231) 